\title{
Treatment of early Pseudomonas aeruginosa infection in patients with cystic fibrosis: the ELITE trial
}

\author{
Felix Ratjen, ${ }^{1}$ Anne Munck, ${ }^{2}$ Pearl Kho, ${ }^{3}$ Gerhild Angyalosi, ${ }^{4}$ for the ELITE Study \\ Group*
}

- Supplementary figures are published online only at http:// thorax.bmi.com/content/vol65/ issue 4

${ }^{1}$ Hospital for Sick Children, Toronto, Canada

${ }^{2}$ Hospital Robert Debre, AP-HP Paris, France

${ }^{3}$ Novartis Horsham Research Centre, Horsham, West Sussex,

${ }^{4}$ Novartis Pharma AG, Basel, Switzerland

\section{Correspondence to}

Felix Ratjen, Sellers Chair of Cystic Fibrosis, Division of Respiratory Medicine, Department of Pediatrics, University of Toronto, Hospital for Sick Children, 555 University Avenue, Toronto M5G 1X8, Ontario, Canada; felix.ratjen@ sickkids.ca

ELITE study collaborators: Germany: Professor Ratjen (Essen), Dr Ballmann (Hannover), Professor Dockter (Hamburg), Dr Mainz (Jena), Dr Nusslein (Bochum), Dr Posselt (Frankfurt), Dr Rietschel (Cologne), Dr Leichsenring (Ulm), Dr Staab (Berlin); France: Dr Munck, Dr Gerardin, Dr Delaisi (Paris-Debre), Dr Fayon (Bordeaux), Professor Ginies (Angers), Dr Le Bourgeois (Paris-Necker), Professor Marguet (Rouen), Professor Roussey (Rennes); The Netherlands: Dr Tiddens (Rotterdam), Dr Bert Arets (Utrecht): UK: Dr Spencer (Newcastle upon Tyne), Dr Weller (Birmingham); Spain: Dr Cobos (Barcelona); Austria: Professor Eichler (Vienna)

* ELITE investigators are listed as collaborators at the end of the manuscript.

Received 15 June 2009 Accepted 27 October 2009 Published Online First 8 December 2009

\section{ABSTRACT}

Rationale Antibiotic therapy for early Pseudomonas aeruginosa infection in patients with cystic fibrosis (CF) is effective, but the optimal therapeutic regimen and duration for early treatment remains unclear. The Early Inhaled Tobramycin for Eradication (ELITE) study was designed to assess the efficacy and safety of two regimens (28 and 56 days) of tobramycin inhalation solution (TIS) $300 \mathrm{mg} / 5 \mathrm{ml}$ twice daily for the treatment of early onset $P$ aeruginosa infection in patients with CF. Methods In this open-label randomised multicentre study, patients with CF (aged $\geq 6$ months) with early $P$ aeruginosa infection were treated for 28 days with TIS twice daily administered by the PARI LC PLUS (PARI $\mathrm{GmbH}$, Starnberg, Germany) jet nebuliser. After 28 days, patients were randomised 1:1 to either stop TIS ( $\mathrm{n}=45$ ) or to receive a further 28 days of TIS $(n=43)$. The primary endpoint was the median time to recurrence of $P$ aeruginosa (any strain). Secondary endpoints included the proportion of patients free of $P$ aeruginosa infection 1 month after cessation of therapy and safety assessments.

Results The median time to recurrence of $P$ aeruginosa (any strain) was similar between the two groups. In total, $93 \%$ and $92 \%$ of the patients were free of $P$ aeruginosa infection 1 month after the end of treatment and $66 \%$ and $69 \%$ remained free at the final visit in the 28-day and 56-day groups, respectively. TIS was well tolerated.

Conclusions Treatment with TIS for 28 days is an effective and well tolerated therapy for early $P$ aeruginosa infection in patients with CF. Trial registration number NCT00391976.

\section{INTRODUCTION}

Cystic fibrosis (CF) is a hereditary disorder that significantly reduces a patient's life expectancy. Individuals with CF are highly susceptible to early endobronchial infections and chronic Pseudomonas aeruginosa infection has a major negative impact on the course of lung disease in these individuals. ${ }^{1} 2$ Once chronic infection with a mucoid phenotype of $P$ aeruginosa is established, eradication is rarely possible even with intense treatment. Studies have shown, however, that antibiotic therapy initiated shortly after a new detection of $P$ aeruginosa is effective in preventing or delaying the onset of chronic infection. ${ }^{3-6}$ This is now considered the standard of care in European CF centres. ${ }^{7} 8$

Antibiotics administered via parenteral, inhaled and oral routes are efficacious; however, the optimal regimen and duration of therapy remains unclear. Inhaled antibiotics are an attractive option, delivering high concentrations of antibiotic directly to the infection site while minimising systemic exposure. ${ }^{910}$ Several small studies have shown that eradication of $P$ aeruginosa infection can be achieved with early inhaled tobramycin treatment. ${ }^{611} 12$ A bronchoalveolar lavage-based study using 28 days of tobramycin inhalation solution (TIS) $300 \mathrm{mg} / 5 \mathrm{ml}$ (TOBI; Novartis Pharma AG, Basel, Switzerland) twice daily suggested a high rate of efficacy whereas previous studies have used lower doses of tobramycin and longer treatment periods. ${ }^{5} 611$ These positive results have prompted further large-scale investigations into the use of inhaled tobramycin. The present EarLy Inhaled Tobramycin for Eradication (ELITE) trial was designed to assess the short- and long-term efficacy and safety of TIS $300 \mathrm{mg} / 5 \mathrm{ml}$ twice daily for 28 and 56 days in the treatment of early onset $P$ aeruginosa infection in patients with CF.

\section{METHODS}

Patients who regularly attended outpatient clinics, had microbiological testing at least every 3 months and a first or early positive $P$ aeruginosa culture at a routine hospital visit were eligible for enrolment. Patients were enrolled from 21 centres in Germany, France, The Netherlands, UK, Spain and Austria.

\section{Study design and patients}

This was an open-label two-arm randomised multicentre study (figure 1) conducted between November 2003 and January 2008. Patients aged $\geq 6$ months with confirmed CF (clinical signs consistent with CF and a sweat chloride $>60 \mathrm{mEq} / \mathrm{l}$ by quantitative pilocarpine iontophoresis or genotype with two identifiable mutations consistent with (F) and a first or early infection with $P$ aeruginosa were eligible. Early infection was defined as a new detection of $P$ aeruginosa in a respiratory culture after negative cultures for at least 1 year if at least four documented negative cultures were available or up to 2 years with four documented negative cultures in this time period in the absence of anti-pseudomonal treatment. Patients were excluded if they had a history of aminoglycoside hypersensitivity or adverse reaction to inhaled aminoglycosides; signs or symptoms of acute pulmonary disease (eg, pneumonia, pneumothorax); a history of hearing loss; had received any investigational drug within 30 days, loop diuretics within 7 days before enrolment, or macrolide antibiotics as a maintenance therapy for $\geq 12$ days during the 28 days before visit 1 (screening/baseline).

All patients received TIS twice daily for 28 days administered via PARI LC PLUS jet nebuliser and a suitable compressor (pressure and flow operating 


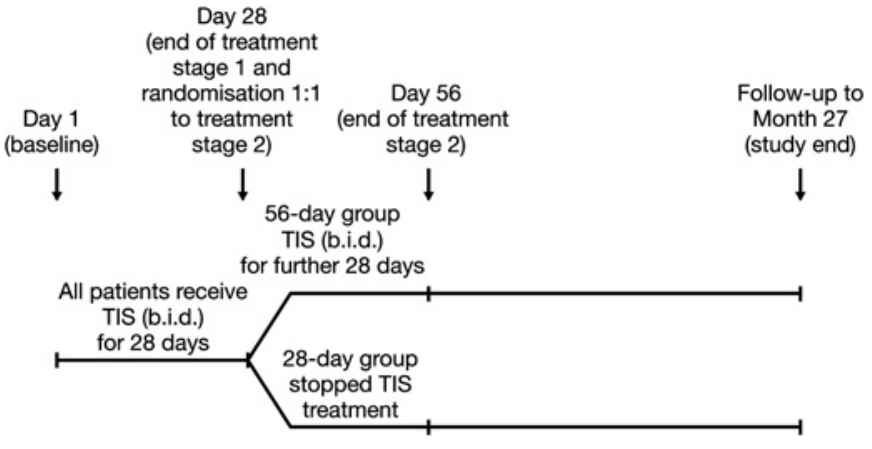

Figure 1 Trial design. TIS, tobramycin inhalation solution $300 \mathrm{mg} / 5 \mathrm{ml}$.

parameters of $110-217 \mathrm{kPa}$ and $4-61 / \mathrm{min}$, respectively). Randomisation occurred at day 28. Patients were excluded from randomisation if antibody titres assessed with a sensitive radioimmunoassay ${ }^{13}$ analysed in a central laboratory using a blood sample taken at day $11 \mathrm{~h}$ after administration of the first does of inhaled tobramycin for any of the three $P$ aeruginosa exoenzymes (exotoxin A, alkaline protease or elastase) were $\geq 1000$ or serum creatinine levels exceeded the upper normal limit for age. ${ }^{13}$ Eligible patients at day 28 were randomised $1: 1$ to either stop study medication (28-day group) or to receive an additional 28 days of treatment (56-day group). Randomised patients had regular study visits (monthly for the first year then every 3 months thereafter) until a positive $P$ aeruginosa sample was obtained, at which point they could enter a follow-up phase with their routine clinic visits and treatment according to their physician's discretion. Patients who were not randomised (non-randomised group) were treated according to their physician's discretion.

\section{Assessments}

The primary outcome was the median time to recurrence of any strain of $P$ aeruginosa. Secondary outcomes included the proportion of patients free of $P$ aeruginosa 1 month after the end of treatment; time to recurrence of any strain of $P$ aeruginos $a$ by sample collected (deep throat swab or sputum); the number of patients with the same genotype of $P$ aeruginosa at baseline and recurrence or a new genotype at recurrence; the proportion of patients free of $P$ aeruginosa 1 month after the end of treatment for sputum and non-sputum producers and by baseline characteristics (age $(<6,6-18$ or $>18$ years), lung function (forced expiratory volume in $1 \mathrm{~s}\left(\mathrm{FEV}_{1}\right)<80 \%$ or $\geq 80 \%$ predicted $)$ and infection status (first or early infection)); and the number and length of hospital admissions for respiratory indications.

Exploratory outcomes included occurrence of non- $P$ aeruginosa pathogens; changes in $\mathrm{FEV}_{1}$, forced vital capacity and forced expiratory flow between $25 \%$ and $75 \%\left(\mathrm{FEF}_{25-75}\right)$; and changes in the normal development of the patient including weight, height and body mass index.

Safety assessments included adverse events (AEs), clinical laboratory evaluations and audiology. All AEs were recorded throughout the study and the severity and relationship to the study drug were evaluated. Serum tobramycin and serum creatinine levels were monitored using blood samples collected at baseline, on days 28 and 56 and months 15 and 27 (creatinine only).

\section{Statistical analysis}

The target sample size was modelled to provide precision for the measurement of time to recurrence of any strain of $P$ aeruginosa infection (the primary outcome). Sample size estimates were based on simulations using 50-75 patients per treatment group and a median time to recurrence of 6-9 months for the 28-day group and 9-12 months for the 56-day group, assuming an $80 \%$ power to detect a treatment difference with a two-sided significance level of 0.0491 . It was estimated that 120 patients would need to be enrolled in order for 100 patients to be randomised.

For the primary outcome (median time to recurrence of any strain of $P$ aeruginosa), the efficacy evaluable population was used. This included patients who received at least one dose of study medication, were randomised, had documented $P$ aeruginosa infection at screening and microbiological assessments at 1 month after their last dose of TIS, with the following exceptions: no eradication at 1 month after their last dose of TIS, protocol deviation or use of prohibited medications. For other efficacy analyses the safety population was used, which included all patients who received at least one dose of study medication.

For the primary outcome, time to recurrence of $P$ aeruginosa was estimated by the Kaplan-Meier method using $t=0$ at 1 month following the end of treatment in each group. A logrank test was used to detect differences between treatment groups and the relative risk of recurrence and 95\% CIs were estimated using a Cox proportional hazard model. Comparisons between the treatment groups for the other efficacy variables were analysed using the Cochran-Mantel-Haenszel test. Spirometry variables, AEs and other safety variables were analysed with descriptive statistics.

\section{RESULTS}

\section{Baseline demographic and clinical characteristics}

A total of 123 patients were recruited into the study. Of these, 88 were randomised (45 in the 28-day TIS group and 43 in the 56-day TIS group; figure 2) and 65 were included in the efficacy evaluable population (34 and 31 in the 28-day and 56-day TIS groups, respectively). Thirty-five patients were not randomised, 31 because of high $P$ aeruginosa antibody titres and four for other reasons (figure 2). The baseline demographic and clinical characteristics were similar between the two treatment groups (table 1). The mean (SD) overall compliance with treatment was 97.24 (8.21) for patients in the 28-day TIS group and 95.66 (11.64) for patients in the 56-day TIS group. Adherence to treatment based on returned ampoules indicated that over $93 \%$ of patients in both groups were at least $80 \%$ compliant with treatment.

\section{Efficacy}

The proportion of patients free of $P$ aeruginosa at day 28 and 1 month after the end of treatment was comparable in both groups (figure 3 ). The proportion of patients free of $P$ aeruginosa 1 month after the end of treatment was similar in sputum producers and non-sputum producers (figure 4). Patients' age, baseline lung function and baseline infection status did not influence the findings (data not shown).

Sixty-five patients (34 and 31 in the 28-day and 56-day TIS groups, respectively) could be included in the evaluation of the primary outcome (time to recurrence of $P$ aeruginosa). The median time to recurrence was 26.12 and 25.82 months following TIS for 28 and 56 days, respectively, and differences between groups were not significant $(p=0.593$; HR $0.81,95 \% \mathrm{CI}$ 0.37 to 1.75; figure 5). Similar results were observed for deep throat swab and sputum samples (figures E1 and E2 in the online supplement). At the time of each patient's final study visit, $66 \%$ of patients remained free of $P$ aeruginosa in the 28-day TIS group $(n=41)$ and $69 \%$ remained free of $P$ aeruginosa in the 56-day TIS group $(\mathrm{n}=36)$.

Paired samples (baseline and recurrence) were available in 21 patients, of which 12 had the same genotype at baseline and at 
Figure 2 Patient disposition. TIS, tobramycin inhalation solution $300 \mathrm{mg} / 5 \mathrm{ml}$. Inappropriate enrolment includes having positive antibody titres for any of the three Pseudomonas aeruginosa exoenzymes, abnormal creatinine levels, an abnormal audiology test or negative for $P$ aeruginosa at baseline.

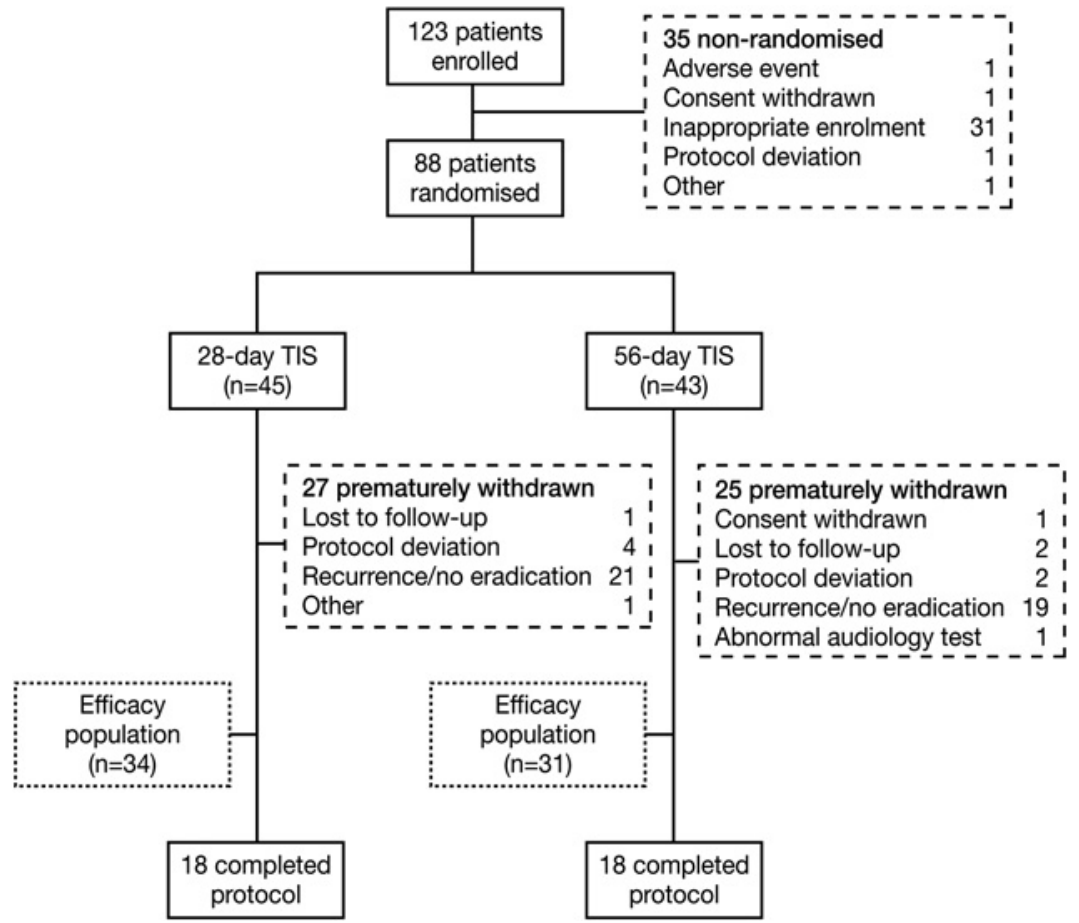

recurrence. For the remaining patients $(n=9)$, paired samples were of a different genotype. There were no consistent trends in the emergence of non-P aeruginosa organisms (table 2 ). Two patients $(5.3 \%)$ in the 56 -day TIS group were hospitalised on one occasion, each for a pulmonary exacerbation during the study (ie, until recurrence of $P$ aeruginosa) although neither was receiving treat-

Table 1 Baseline demographic and clinical characteristics (all enrolled population)

\begin{tabular}{|c|c|c|}
\hline & 28-day TIS $(n=45)$ & 56-day TIS $(n=43)$ \\
\hline Mean (SD) age (years) & $8.7(7.2)$ & $8.7(10.5)$ \\
\hline \multicolumn{3}{|l|}{ Age group, $\mathrm{n}(\%)$} \\
\hline 6 months $-<6$ years & $19(42)$ & $18(42)$ \\
\hline $6-<18$ years & $20(44)$ & $21(49)$ \\
\hline$\geq 18$ years & $6(13)$ & $4(9)$ \\
\hline \multicolumn{3}{|l|}{ Gender, n (\%) } \\
\hline Female & $19(42)$ & $21(49)$ \\
\hline Male & $26(58)$ & $22(51)$ \\
\hline Mean (SD) weight (kg) & $30.0(19.8)$ & $27.4(17.3)$ \\
\hline Mean (SD) height (cm) & $124.8(34.3)$ & $121.9(31.7)$ \\
\hline Previously vaccinated against $\mathrm{Pa}, \mathrm{n}(\%)$ & $2(4)$ & $7(16)$ \\
\hline \multicolumn{3}{|l|}{ Cystic fibrosis genotype, n (\%) } \\
\hline F508/F508 & $17(38)$ & $21(49)$ \\
\hline F508/other & $19(42)$ & $14(33)$ \\
\hline Other/other & $5(11)$ & $4(9)$ \\
\hline Unknown/not available & $4(9)$ & $4(9)$ \\
\hline \multicolumn{3}{|l|}{ Infection with $\mathrm{Pa}, \mathrm{n}(\%)$} \\
\hline First & $28(62)$ & $27(63)$ \\
\hline Early, after 1 year negative culture & $8(18)$ & $8(19)$ \\
\hline Early, after 2 years negative culture & $8(18)$ & $8(19)$ \\
\hline Not available & $1(2)$ & $0(0)$ \\
\hline \multicolumn{3}{|l|}{ Pulmonary function tests, mean (SD) } \\
\hline $\mathrm{FEV}_{1} \%$ predicted & $80.2(18.9)$ & $87.0(19.2)$ \\
\hline FVC \% predicted & $91.1(15.3)$ & $99.2(20.2)$ \\
\hline $\mathrm{FEF}_{25-75} \%$ predicted & $61.5(28.8)$ & $63.4(26.9)$ \\
\hline
\end{tabular}

$\mathrm{FEF}_{25-75}$, forced expiratory flow between $25 \%$ and $75 \%$; $\mathrm{FEV}_{1}$, forced expiratory volume in $1 \mathrm{~s}$; FVC, forced vital capacity; Pa, Pseudomonas aeruginosa; TIS, tobramycin inhalation solution $300 \mathrm{mg} / 5 \mathrm{ml}$. ment at the time of the exacerbation. One patient was hospitalised for 1 day for bronchitis and the other patient was hospitalised for $P$ aeruginosa exacerbation. This patient was treated with intravenous ceftazidime and tobramycin for 14 days.

No major short- or long-term changes in spirometric parameters were observed during the study period (table 3 ). Treatment body mass index of patients in any of the age groups (data not shown).

\section{Safety}

Treatment-emergent AEs (TEAEs) occurring up to month 3 were reported by $32(73 \%), 25(58 \%)$ and $7(20 \%)$ patients in the 28-day TIS, 56-day TIS and the non-randomised groups, respectively (table 4). TEAEs occurring after month 3 were reported by $21(60 \%)$ and $19(53 \%)$ patients in the 28-day and 56-day TIS groups, respectively (table 4). Overall, cough was the most commonly reported TEAE. AEs up to month 3 that were considered possibly or probably related to treatment were reported by 14 patients in each TIS group, with the majority

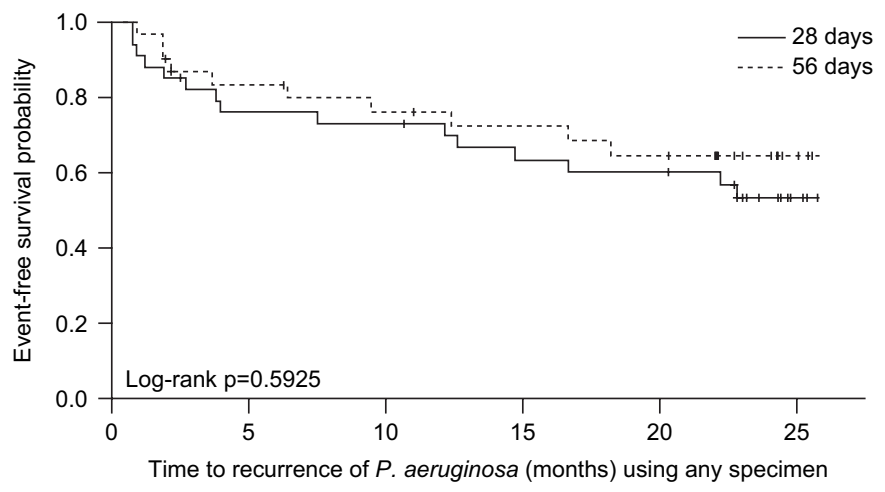

Figure 3 Kaplan-Meier plot of time to recurrence of any strain of Pseudomonas aeruginosa in patients with cystic fibrosis treated with tobramycin inhalation solution twice daily (efficacy evaluable population). with TIS for 28 and 56 days did not influence weight, height or 


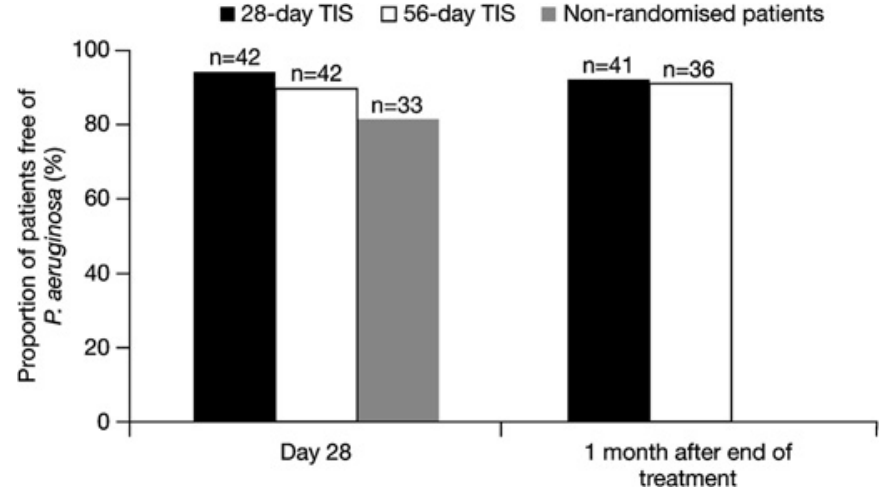

Figure 4 Proportion of patients free of Pseudomonas aeruginosa at day 28 and 1 month after the end of treatment (month 3; safety population). At 1 month after the end of treatment, some patients did not have data available. TIS, tobramycin inhalation solution $300 \mathrm{mg} / 5 \mathrm{ml}$.

being related to dysphonia in both treatment groups $(11 \%$ and $14 \%$, respectively) and cough in the 28-day group (9\%). After month 3 , AEs considered possibly or probably related to treatment were reported by one patient (3\%) in each treatment group (haemoptysis and persistence of Pseudomonas infection).

Six patients $(14 \%)$ in the 28 -day TIS group and five (12\%) in the 56-day TIS group reported serious AEs, four of which were related to infections (Pseudomonas infection $(\mathrm{n}=2)$ and Stenotrophomonas infection $(\mathrm{n}=1)$ in the 28-day TIS group and varicella $(n=1)$ in the 56-day TIS group). One patient in each treatment group withdrew from the study because of an $\mathrm{AE}$ (Pseudomonas infection and no eradication of Pseudomonas). There were no deaths.

No significant changes in serum creatinine concentrations were observed in either group. In the 56-day TIS group, there was no difference between serum tobramycin levels at days 28 and 56 (mean (SD; minimum-maximum) $1.4(1.2 ; 0-5.8) \mu \mathrm{g} /$ $\mathrm{ml}$ vs $1.3(1.1 ; 0-3.7) \mu \mathrm{g} / \mathrm{ml})$ suggesting no accumulation of tobramycin over time. In addition, the serum tobramycin levels were not influenced by the age of the patients. There were two cases of an AE 'drug level increased' reported in the 28-day TIS group. Neither of the cases was serious; one was reported to be mild and the other moderate in severity. Both cases were reported as probably related to study medication. No action was taken and both patients recovered.

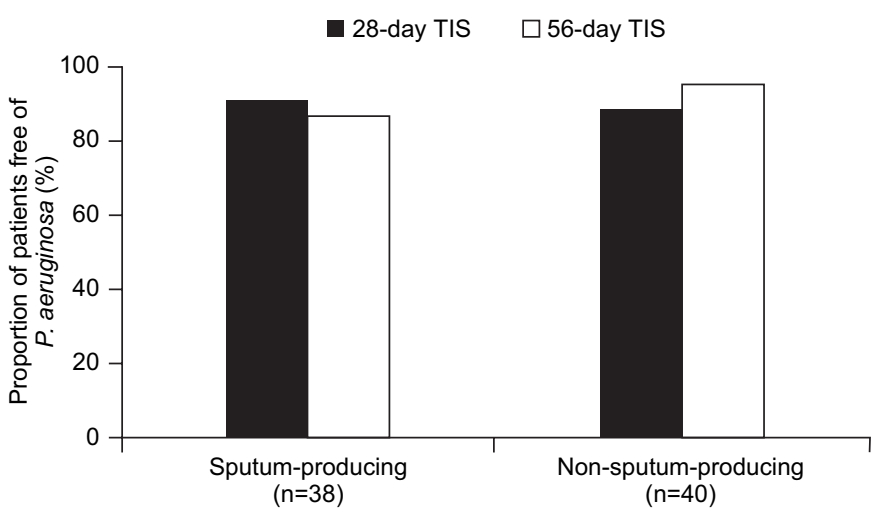

Figure 5 Proportion of sputum-producing and non-sputum-producing patients free of Pseudomonas aeruginosa 1 month after the end of treatment (safety population). TIS, tobramycin inhalation solution $300 \mathrm{mg} / 5 \mathrm{ml}$.
None of the 65 patients (53\% of the safety population) with audiology measurements exhibited ototoxicity, defined as a bilateral increase of $\geq 15 \mathrm{~dB}$ in hearing threshold at two consecutive frequencies between 2 and $8 \mathrm{kHz}$. There were, however, minor increases measured sporadically in two patients in the 56-day group which were reported as an $\mathrm{AE}$ of 'deafness' or hearing loss, one mild and one moderate-to-severe in severity. Both events were reported as probably related to study medication, but no action was taken and both patients' hearing returned to normal.

\section{DISCUSSION}

The ELITE trial shows that treatment with TIS for 28 days is effective for treating early $P$ aeruginosa infection, and extending TIS treatment to 56 days does not provide notable additional improvements. Over $90 \%$ of randomised patients in the 28 -day and 56-day TIS groups had negative cultures for $P$ aeruginosa 1 month after the end of treatment, and the majority of these patients remained free from infection for up to 27 months. The results were similar in sputum and non-sputum producers and were unaffected by the patient's baseline characteristics (age, lung function and first versus recurrent infection).

Antibiotics, either alone or in combination, have been shown to be effective in the treatment of early $P$ aeruginosa infection. ${ }^{3-611} 14$ However, to date there have been no reports of comparative studies assessing different treatment regimens and only a few controlled studies have been performed. In an open controlled trial, inhaled colistin and oral ciprofloxacin twice daily for 3 weeks was shown to delay chronic $P$ aeruginosa infection in patients with CF. ${ }^{4}$ Extension of the treatment period to 3 months has been shown to provide additional benefit. ${ }^{15}$ This treatment regimen has been adopted by other centres and effectively reduced the rate of chronic $P$ aeruginosa infection as well as treatment cost. ${ }^{16-18}$

In previous studies, including a placebo-controlled trial of antibiotic monotherapy, early treatment with inhaled tobramycin $80 \mathrm{mg}$ twice daily for 12 months was shown to have a success rate similar to that seen with combination therapy. ${ }^{4-6}$ A further study showed that TIS $300 \mathrm{mg}$ twice daily for 28 days resulted in negative bronchoalveolar lavage cultures at the end of treatment in all eight patients receiving tobramycin, whereas $P$ aeruginosa density was unchanged with placebo. ${ }^{11}$ This was supported by a subsequent study showing that TIS monotherapy for 28 and 56 days eradicated lower airway $P$ aeruginosa for up to 3 months. ${ }^{12}$ It should be noted that patients with a history of $P$ aeruginosa infection within the last 12 months were included in these studies. In contrast, the ELITE study, similar to other European studies on this topic, focused on patients with first or early infection. Treatment success rates in this trial compare favourably with previous studies. The ELITE trial also demonstrated long-term efficacy and further supports the use of inhaled tobramycin alone as a successful treatment strategy for early $P$ aeruginosa infection.

Inhaled antibiotics may not reach all areas of the lung due to mucus plugs obstructing the airways, and the addition of systemic therapy may increase the chances of eradication. Deposition of antibiotics is less homogeneous in patients with more advanced disease, and this population may benefit from a combined treatment approach. In the ELITE trial, efficacy was similar in patients with normal versus reduced lung function $\left(\mathrm{FEV}_{1}\right)$ at baseline, and age did not influence the treatment effect. The overall success rate of treatment was high and compares favourably with results for other regimens. ${ }^{4-6} 1114$ 
Table 2 Isolation frequency of non-Pseudomonas aeruginosa organisms at baseline and months 3 and 27 (efficacy evaluable population)

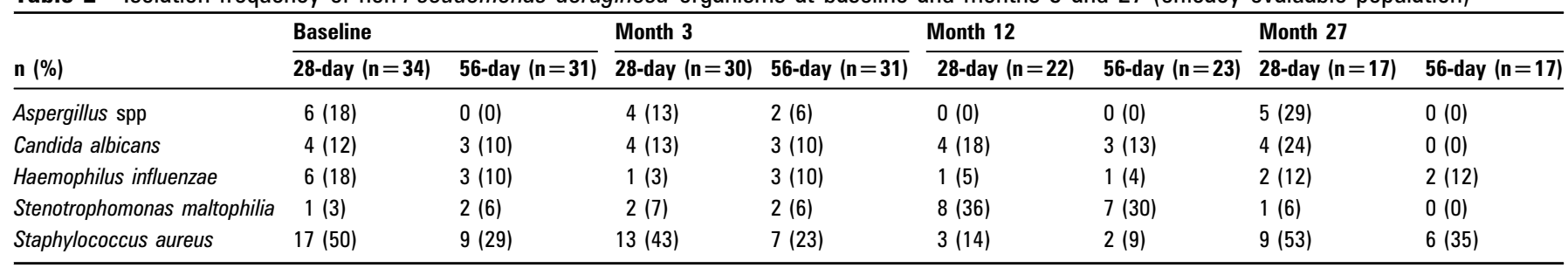

Head-to-head comparisons of inhaled antibiotics alone versus a combination of inhaled antibiotics with oral ciprofloxacin have not been reported. However, the Early Pseudomonas Infection Control study is currently ongoing and will compare different treatment strategies, including the combination of TIS with oral ciprofloxacin versus TIS alone. ${ }^{19}$

Accurate diagnosis of lower airway infection is challenging in younger children and patients with milder disease as they often do not expectorate sputum. In such cases, throat or cough swabs are used. Studies comparing bronchoalveolar lavage with throat swabs have shown a good negative but low positive predictive value of these swabs. ${ }^{10}$ This suggests that throat swabs are most useful in excluding lower airway infection in CF. Treatment decisions based solely on throat swabs may lead to overtreatment as the low positive predictive may overestimate the proportion of patients with $P$ aeruginosa in their lower respiratory tract. The presence of Paeruginosa in the upper airway may, however, convey a risk for subsequent lower airway infection. Although this sequence of events is under debate, placebocontrolled studies have shown that the majority of untreated patients with a positive throat swab for Paeruginosa will develop chronic infection. ${ }^{20}$ This therefore favours aggressive treatment strategies where any respiratory culture is positive for $P$ aeruginosa. In the ELITE trial, approximately $50 \%$ of the patients were sputum producers and it is reassuring that the efficacy was similar in both sputum-producing and non-sputum-producing patients.

Similar to other studies, the ELITE trial used a microbiological and not a clinical outcome as the primary endpoint. Chronic $P$ aeruginosa infection is associated with a more rapid decline in lung function, and eradication of the pathogen may reverse this process. Limited data suggest that, compared with patients without infection, those with $P$ aeruginosa infection have reduced lung function even after successful eradication of the pathogen. ${ }^{21}$ In the ELITE trial, lung function remained stable throughout the study but, given the overall slow rate of decline in $\mathrm{FEV}_{1}$ in patients with $\mathrm{CF}$, a longer follow-up is required to assess the long-term effects of treatment of early $P$ aeruginosa infection on lung function decline. Ideally, a long-term placebocontrolled study should be conducted but, given that treatment of early $P$ aeruginosa infection is now standard practice in most CF centres, ${ }^{7}$ such a study is unfeasible. Results from observational studies will therefore be needed to help define the longterm disease course of patients after $P$ aeruginosa eradication.

Table 3 Median change from baseline to months 3 and 27 for spirometry parameters (combined 28-day and 56-day TIS groups)

\begin{tabular}{lrr}
\hline & Month 3 & \multicolumn{1}{c}{ Month 27 } \\
\hline FEV $_{1} \%$ predicted & $0.6(\mathrm{n}=39)$ & $-1.3(\mathrm{n}=18)$ \\
FVC \% predicted & $-2.7(\mathrm{n}=39)$ & $-2.3(\mathrm{n}=18)$ \\
$\mathrm{FEF}_{25-75} \%$ predicted & $-0.1(\mathrm{n}=36)$ & $0.2(\mathrm{n}=17)$ \\
\hline $\mathrm{FEF}_{25-75,}$ forced expiratory flow between $25 \%$ and $75 \% ; \mathrm{FEV}_{1}$, forced expiratory volume in \\
1 s; FVC, forced vital capacity; TIS, tobramycin inhalation solution.
\end{tabular}

It should be noted that the sample size in the ELITE study was lower than planned. The study did, however, meet its primary objective to determine a valid point estimate of the treatment effect on time to recurrence for the 28-day and 56-day treatment regimens. There were no differences between the two treatment groups and, moreover, the recurrence rate observed was lower than initial assumptions based on findings from previous studies. A post hoc sample size calculation based on the study results showed that 1368 patients would have to enter a trial to show a potential difference in treatment efficacy with $80 \%$ power, which makes it likely that even a considerably larger sample size may only be able to show a small and probably clinically insignificant difference, if any, between the treatment regimens.

Treatment with TIS was well tolerated. Only a few serious AEs or discontinuations due to AEs were reported and most appeared to be related to CF rather than the study drug. The study supports previous data that inhaled antibiotic therapy has a high safety margin and is unlikely to cause nephrotoxicity and ototoxicity that is seen after parenteral administration of aminoglycosides. ${ }^{22}$ The two events of hearing loss reported, given their transient nature and spontaneous recovery, are of uncertain clinical significance. Importantly, no accumulation of tobramycin was observed even in young children and the lack of an age dependency in serum levels indicates that the same dose could be used from infancy to adulthood. Data for infants $<6$ months of age are still sparse, but the overall frequency of developing $P$ aeruginosa infection in these young infants is relatively low.

Airway infection in patients with CF can be caused by multiple organisms and treatment of $P$ aeruginosa could promote airway infection with other organisms. The ELITE trial used regular monthly monitoring of respiratory cultures in the first year to capture the emergence of any non- $P$ aeruginosa pathogens. No obvious trends in emerging pathogens were observed, which supports the safety of this treatment approach.

Currently, there are no consistent guidelines for defining early, intermittent and chronic $P$ aeruginosa infection, which mainly relies on detection of the pathogen alone or in combination with antibody testing. The ELITE trial used strict microbiological criteria along with antibody detection to exclude patients with longstanding $P$ aeruginosa infection. Treatment efficacy appeared to be relatively high in patients excluded on the basis of positive antibodies, but long-term follow-up of these patients was not assessed in this trial. The patient selection process was intended to mimic clinical practice where $P$ aeruginosa is usually first detected during a routine clinic visit. Further studies are required to determine whether or not this treatment regimen will be efficacious in patients with newly acquired $P$ aeruginosa associated with clinical signs and symptoms of a pulmonary exacerbation.

In conclusion, TIS treatment for 28 and 56 days in patients aged $\geq 6$ months is both effective and well tolerated in the treatment of early $P$ aeruginosa infection with CF. The results of 
Table 4 Most common treatment-emergent adverse events (occurring in $\geq 5 \%$ in any group; safety population)

\begin{tabular}{|c|c|c|c|c|c|}
\hline & \multicolumn{2}{|l|}{ 28-day TIS } & \multicolumn{2}{|l|}{ 56-day TIS } & \multirow{2}{*}{$\begin{array}{l}\text { Non-randomised } \\
\text { Before month } 3(n=35)\end{array}$} \\
\hline & Before month $3(n=44)$ & After month $3(n=35)$ & Before month $3(n=43)$ & After month $3(n=36)$ & \\
\hline Any adverse event & $32(73)$ & $21(60)$ & $25(58)$ & $19(53)$ & $7(20)$ \\
\hline Rhinitis & $7(16)$ & $3(9)$ & $3(7)$ & $2(6)$ & $1(3)$ \\
\hline Dysphonia & $5(11)$ & 0 & $6(14)$ & 0 & $1(3)$ \\
\hline URTI & $4(9)$ & $4(11)$ & $2(5)$ & $4(11)$ & 0 \\
\hline Pyrexia & $3(7)$ & $2(6)$ & $4(9)$ & $2(6)$ & 0 \\
\hline Haemoptysis & $2(5)$ & $1(3)$ & $1(2)$ & 0 & 0 \\
\hline Lung disorder & 2 (5) & $1(3)$ & 0 & 2 (6) & 0 \\
\hline Oropharyngeal pain & 2 (5) & $2(6)$ & 0 & 0 & 0 \\
\hline Bronchitis & $2(5)$ & $3(9)$ & 0 & $4(11)$ & 0 \\
\hline Influenza & $2(5)$ & 0 & 0 & 0 & 0 \\
\hline Vomiting & $1(2)$ & 0 & $1(2)$ & $3(8)$ & 0 \\
\hline Otitis media & 0 & $3(9)$ & $1(2)$ & $2(6)$ & 0 \\
\hline Sinusitis & 0 & $2(6)$ & 0 & 0 & 0 \\
\hline Deafness & 0 & 0 & $2(5)$ & 0 & 0 \\
\hline Varicella & 0 & 0 & $1(2)$ & $3(8)$ & 0 \\
\hline
\end{tabular}

Values shown are $\mathrm{n}(\%)$

No adverse events were reported in the non-randomised group after month 3 .

TIS, tobramycin inhalation solution $300 \mathrm{mg} / 5 \mathrm{ml}$; URTI, upper respiratory tract infection.

this study compare favourably with others using longer treatment regimens or combinations with oral ciprofloxacin. Headto-head comparisons will clarify whether adding either oral ciprofloxacin or intravenous antibiotic therapy can further increase treatment success in patients with CF with early $P$ aeruginosa infection.

Acknowledgements The authors thank Professor E Bingen for conducting the genotypic pulse-field strain $P$ aeruginosa analysis. Editorial assistance was provided by Clare Wheatcroft. This assistance was funded by Novartis Pharma AG.

Funding Chiron was involved in the initiation and design of the study. Novartis Pharma AG, Basel, Switzerland, subsequently assumed sponsor's responsibility and was involved in the collection, analysis and interpretation of data.

Competing interests PK and GA work for Novartis, the sponsor of this trial.

Ethics approval This study was approved by the ethics committees or institutional review boards at each centre and conducted in accordance with the Declaration of Helsinki and Good Clinical Practice according to the International Conference on Harmonisation guidelines. Each patient and/or their parent or legal guardian gave written informed consent.

Provenance and peer review Not commissioned; externally peer reviewed.

\section{REFERENCES}

1. Emerson J, Rosenfeld M, McNamara S, et al. Pseudomonas aeruginosa and other predictors of mortality and morbidity in young children with cystic fibrosis. Pediatr Pulmonol 2002;34:91-100.

2. Kosorok MR, Zeng L, West SE, et al. Acceleration of lung disease in children with cystic fibrosis after Pseudomonas aeruginosa acquisition. Pediatr Pulmonol 2001:32:277-87.

3. Littlewood JM, Miller MG, Ghoneim AT, et al. Nebulised colomycin for early pseudomonas colonisation in cystic fibrosis. Lancet 1985:13:865.

4. Valerius NH, Koch C, Høiby N. Prevention of chronic Pseudomonas aeruginosa colonisation in cystic fibrosis by early treatment. Lancet 1991:338:725-6.

5. Wiesemann HG, Steinkamp G, Ratjen F, et al. Placebo-controlled, double-blind randomized study of aerolized tobramycin for early treatment of Pseudomonas aeruginosa colonization in cystic fibrosis. Pediatr Pulmonol 1998;25:88-92

6. Ratjen F, Döring G, Nikolaizik WH. Effect of inhaled tobramycin on early Pseudomonas aeruginosa colonisation in patients with cystic fibrosis. Lancet 2001;358:983-4.
7. Döring G, Conway SP, Heijerman HGM, et al. Antibiotic therapy against Pseudomonas aeruginosa in cystic fibrosis: a European consensus. Eur Respir J 2000:16:749-67.

8. Döring G, Hoiby N. Consensus Study Group. Early intervention and prevention of lung disease in cystic fibrosis: a European consensus. J Cyst Fibros 2004;3:67-91.

9. Touw DJ, Brimicombe RW, Hodson ME, et al. Inhalation of antibiotics in cystic fibrosis. Eur Respir J 1995;8:1594-604.

10. Rosenfeld M, Cohen M, Ramsey B. Aerosolized antibiotics for bacterial lower airway infections: principles, efficacy and pitfalls. Clin Pulm Med 1997:4:101-12.

11. Gibson RL, Emerson J, McNamara S, et al. Significant microbiological effect of inhaled tobramycin in young children with cystic fibrosis. Am J Respir Crit Care Med 2003;167:841-9.

12. Gibson RL, Emerson J, Mayer-Hamblett N, et al. Duration of treatment effect after tobramycin solution for inhalation in young children with cystic fibrosis. Pediatr Pulmonol 2007;:42:610-23.

13. Döring G, Obernesser $\mathrm{JH}$, Botzenhart K. Extracellular toxins of Pseudomonas aeruginosa. III. Radioimmunoassay for detection of alkaline protease. Zentralb/ Bakteriol Mikrobiol Hyg A 1982;252:239-47.

14. Munck A, Bonascorsi S, Mariani-Kurkdijian P, et al. Genotypic characterization of Pseudomonas aeruginosa strains recovered from patients with cystic fibrosis after initial and subsequent colonization. Pediatr Pulmonol 2001;32:288-92.

15. Frederiksen B, Koch C, Høiby N. Antibiotic treatment of initial colonization with Pseudomonas aeruginosa postpones chronic infection and prevents deterioration of pulmonary function in cystic fibrosis. Pediatr Pulmonol 1997;23:330-5.

16. Lee TW, Brownlee KG, Denton $\mathrm{M}$, et al. Reduction in prevalence of chronic Pseudomonas aeruginosa infection at a regional pediatric cystic fibrosis center Pediatr Pulmonol 2004:37:104-10.

17. Taccetti G, Campana S, Festini F, et al. Early eradication therapy against Pseudomonas aeruginosa in cystic fibrosis patients. Eur Respir J 2005;26 458-61.

18. Hansen CR, Pressler T, Høiby N. Early aggressive eradication therapy for intermitten Pseudomonas aeruginosa airway colonization in cystic fibrosis patients: 15 years experience. J Cyst Fibros 2008; 7:523-30.

19. National Heart, Lung and Blood Institute, Cystic Fibrosis Foundation, CF Therapeutics Development Network Coordinating Center. Comparison of two treatment regimens to reduce PA infection in children with cystic fibrosis (EPIC). Bethesda, Maryland: National Library of Medicine (US), 2004 http://clinicaltrials.gov/ct2/show/NCT00097773 (accessed Jan 2009).

20. Ratjen F. Treatment of early Pseudomonas aeruginosa infection in patients with cystic fibrosis. Curr Opin Pulm Med 2006;12:428-32.

21. Kozlowska WJ, Bush A, Wade A, et al. Lung function from infancy to the preschoo years after clinical diagnosis of cystic fibrosis. Am J Respir Crit Care Med 2008:178:42-9.

22. Kumana CR, Yuen KY. Parenteral aminoglycoside therapy. Selection, administration and monitoring. Drugs 1994;47:902-13. 